\title{
Inflammatory Fibroid Polyp: A Rare Benign Tumor of the Alimentary Tract in Children Presenting As Intussusception- Case Report and Review of Literature
}

\author{
${ }^{1}$ Department of Paediatric Surgery and Urology, Manchester \\ Children's Hospital, Manchester, United Kingdom \\ 2 Department of Paediatric Surgery, Alder Hey Children's Hospital, \\ Liverpool, United Kingdom \\ 3 Department of Paediatric Histopathology, Alder Hey Children's \\ Hospital, Liverpool, United Kingdom
}

Sotirios Siminas ${ }^{1}$ Eyas Qasem ${ }^{2}$ Rajeev Shukla ${ }^{3}$ Richard Turnock ${ }^{2}$

Eur J Pediatr Surg Rep 2014;2:16-19.

\begin{abstract}
Address for correspondence Sotirios Siminas, MD, PhD, FEBPS, Department of Paediatric Surgery and Urology, Manchester Children's Hospital, Oxford Road, Manchester M13 9WL, United Kingdom (e-mail: siminas1@gmail.com).
\end{abstract}

\begin{abstract}
Keywords

- inflammatory

- fibroid

- polyp

- intussusception

Inflammatory fibroid polyp (IFP) represents a rare cause of gastrointestinal polypoid disease in childhood. The lesion has been described by various names beyond the currently accepted term, including "Vanek's tumour," eosinophilic or submucosal granuloma, gastric fibroma with eosinophilic infiltration, inflammatory pseudotumor, and hemangiopericytoma. The etiopathogenesis and origin of the mesenchymal spindle-shaped cells that comprise the polyp remains enigmatic. Recent studies have shown familial occurrence, expression of platelet-derived growth factor receptor (PDGFRA) and oncogenic PDGFRA mutations in the majority of lesions, suggestive of a neoplastic nature. We present a rare case of a 10-year-old boy with an IFP of the terminal ileum, who presented acutely with intussusception and was treated with a right hemicolectomy. Postoperative course was uneventful and the patient has been asymptomatic during follow-up. Histopathology and immunohistochemical analysis excluded inflammatory myofibroblastic tumor (negative for Alk1, desmin, smooth muscle actin [SMA]), gastrointerstinal stromal tumors (GIST) (negative for CD117) and schwannoma (negative for S100). The lesion was positive for CD34 and faintly for vimentin. Despite the classification of IFPs as a mesenchymal benign neoplasm, in the vast majority of cases, surgical excision alone was curative, and no reports exist of a malignant transformation. A cautious approach with periodic surveillance of the affected children seems reasonable though.
\end{abstract}

\section{Introduction}

Inflammatory fibroid polyp (IFP) represents one of the rarest causes of polypoid disease of the gastrointestinal (GI) tract in childhood. It is characterized by submucosal fibrous connective tissue growth with spindle-shaped cells in a hypervascular stroma infiltrated by nonspecific inflammatory cells, especially eosinophils. After its first report by Vanek in $1949,{ }^{1}$ the lesion has been described by various names, including "Vanek's tumour," eosinophilic or submucosal granuloma, gastric fibroma with eosinophilic infiltration, inflammatory pseudotumor, and hemangiopericytoma, with the term IFP (proposed in 1953) currently widely accepted. ${ }^{2}$ The lesions are usually solitary and may be located anywhere across the GI tract. The etiopathogenesis, as well as the origin of the mesenchymal spindle-shaped cells that comprise the polyp received

May 10, 2013

accepted after revision

July 25, 2013

published online

September 9, 2013

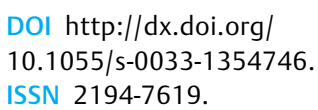

DOI http://dx.doi.org/

$10.1055 / \mathrm{s}-0033-1354746$. ISSN 2194-7619.

(c) 2014 Georg Thieme Verlag KG
Stuttgart · New York

License terms

(c) $(1) \$$ 
remains enigmatic. Recent studies though have shown familial occurrence of the polyps, ${ }^{3}$ expression of platelet-derived growth factor receptor (PDGFRA), and oncogenic PDGFRA mutations in the majority of these tumors, ${ }^{2,4,5}$ suggestive of a neoplastic nature. We present a rare case of a child with an IFP of the terminal ileum, who presented with intussusception.

\section{Case Report}

A 10-year-old boy was referred for a surgical opinion, with worsening symptoms of intermittent abdominal pain associated with drowsiness that started 10 days prior, after a 24hour period of diarrhea. Medical history included some features of Noonan syndrome including short stature for which treatment with growth hormone had been commenced. On presentation, he was hemodynamically stable and well hydrated. Abdominal examination revealed the presence of a tender cylindrical mass in the epigastrium, with no signs of peritonism. Inflammatory markers were normal. Abdominal ultrasound showed the presence of a complex, multilayered mass in the epigastrium, with features suggestive of intussusception. Because of the complex nature of the lesion, a computerized tomography scan of the abdomen was also performed and that showed a complex multilayered cystic and solid mass, approximately $8 \times 5 \mathrm{~cm}$ lying inferior to, compressing and displacing a thick-walled inflamed stomach. Imaging findings could not exclude the presence of an internal hernia, or gastric or small bowel volvulus. Emergency laparotomy was performed, at which an ileocolic intussusception in the epigastrium was found with an intraluminal mass in the terminal ileum as a focal point. The intraoperative impression was that the lesion represented a lymphoma therefore a right hemicolectomy with ileocolic anastomosis was performed. The patient recovered well from surgery.

Histopathology reported the presence of a large polypoid mass with a fleshy red surface in the terminal ileum $(3.5 \times 3 \times 2.5 \mathrm{~cm})$. The polyp showed surface ulceration with granulation tissue on the surface ( - Fig. 1 ), but with the polyp itself arising from the submucosa and being composed of spindle cells in a loose fibromyxoid stroma. The stroma had rich inflammatory mainly eosinophilic infiltrate and thin collagen bundles with variably ectatic blood vessels (-Fig. 2). There was no atypia or mitotic features. Immunohistochemistry excluded inflammatory myofibroblastic tumor (negative for Alk1, desmin, SMA), GIST (negative for CD117), and schwannoma (negative for S100). The lesion was positive for $\mathrm{CD} 34$ and faintly for vimentin. Overall, the appearances were typical of an IFP, completely excised. Cytogenetic studies with fluorescence in situ hybridization were done to exclude rearrangement of the Alk gene (anaplastic lymphoma kinase) which is identified in inflammatory myofibroblastic tumors and some colorectal carcinomas. Mutations in the PDGFRA gene were not assessed in this analysis.

The patient has been symptom-free after a year of followup, and remains under clinical surveillance.

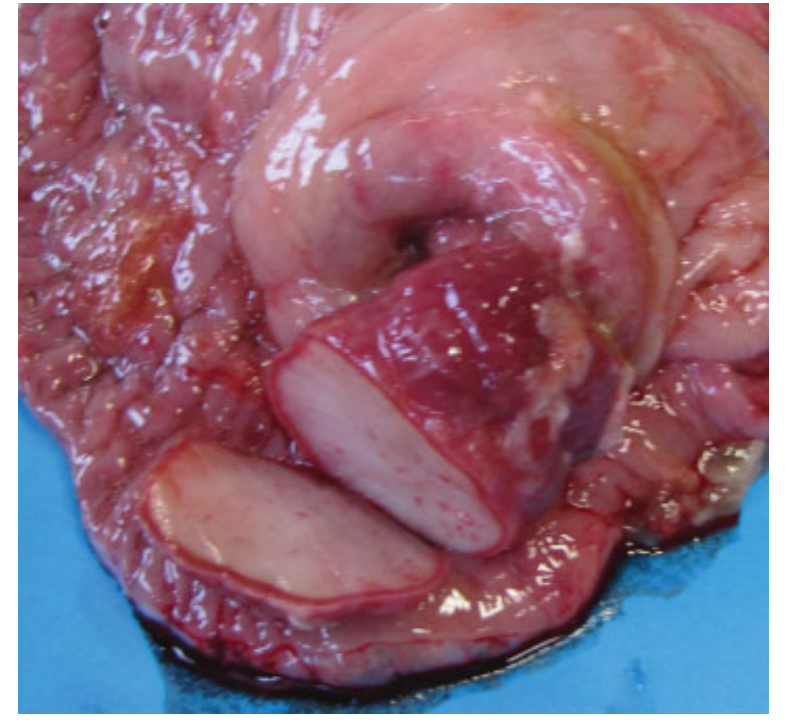

Fig. 1 A polyp, cut open, showing surface ulceration.

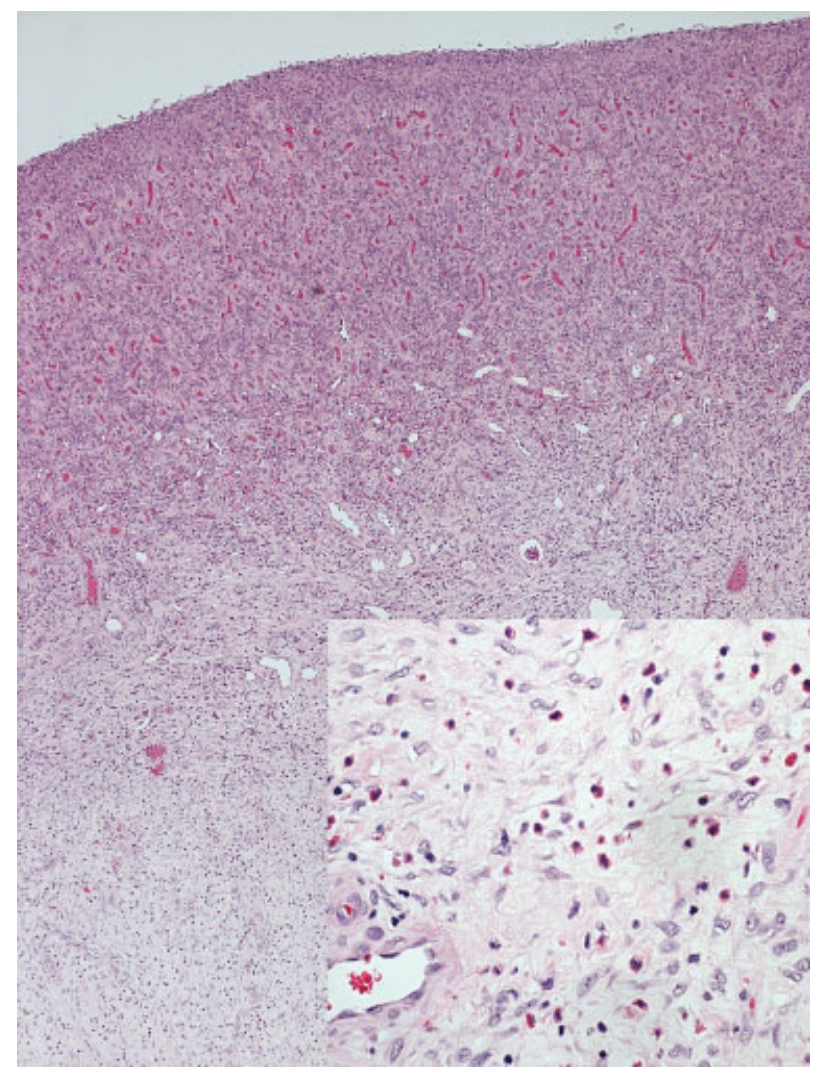

Fig. 2 Lesion is composed of spindle-to-stellate-shaped cells in an oedematous stroma. There is prominent inflammatory infiltrate composed of lymphocytes and eosinophils (Inset right lower corner).

\section{Discussion}

Although these lesions were regarded in the past to be reactive overgrowths of fibrovascular tissue, recent data have shown that they are rather PDGFRA-driven benign neoplasms, as 55 to $65 \%$ of them have been proven to carry mutations in the PDGFRA gene., ${ }^{4,5}$ They are extremely 
uncommon in childhood as they tend to affect patients after the 5 th to 6 th decade of life. ${ }^{6,7}$ A total of 14 cases have been identified in the literature ${ }^{6,8-12}$ (-Table 1). The lesion can affect the whole of the GI tract, with the stomach most commonly involved in adults, but in children the distribution seems to be slightly different with most cases reported to occur in the ileum (five cases), with intussusception as the main presentation (four cases). Other symptoms related to the lesions are generally nonspecific, and the polyps may manifest themselves with anemia caused by occult GI bleeding, abdominal pain or vomiting, and less frequently with rectal bleeding, intestinal obstruction, or failure to thrive. ${ }^{6-8}$ The polyps are virtually impossible to diagnose preoperatively as their imaging features are nonspecific. Even if endoscopy is undertaken, biopsy may be difficult as the lesions are usually sessile arising from the submucosa with surface erosion and granulation. Therefore, diagnosis is usually established after complete excision of the polyp, which is usually considered intraoperatively to be a lymphoma due to the resulting inflammation. Complete excision is almost always therapeutic and the reported recurrences mainly occurred in patients in whom resection was attempted endoscopically. 7,12

Despite various proposed theories, the origin of the polyp's spindle cells remains largely unknown. As eosinophilic infiltration was one of the most constant findings, an allergybased pathogenesis was proposed, and hence, the name eosinophilic granuloma. In most cases, although there is no history of allergy and eosinophilia is present in only $4 \%$ of the patients, while eosinophilic infiltration can be associated with other GI diagnoses, such as eosinophilic gastroenteritis. ${ }^{6}$ Other proposed etiologies included neural hyperplasia, ${ }^{13}$ leiomyomatous origin, ${ }^{14}$ and metabolic disorders similar to Hand-Schuller-Christian disease or Letterer-Siwe syndrome, ${ }^{15}$ although the most common theory was that the polyps formed as an inflammatory granulomatous reaction to a variety of potential gut insults including trauma, infection or reactions to chemicals, initiating the proliferation of fibroblasts, inflammatory, and stromal cells. ${ }^{16}$ The report of familial occurrence with multiple and recurrent IFPs in a family, ${ }^{3}$ as well as the recognition of PDGRFA gene mutations in exons 12 or $18^{2,4,5}$ has changed the perception regarding these polyps, which are now considered to represent true neoplasms. The PDGFRA gene encodes a receptor tyrosine kinase, and activating mutations in exons 12,14 , and 18 have been found in GISTs and possibly represent a potent oncogenetic event. ${ }^{2,4}$ PDGFRA-related GISTs are usually epithelioid and less aggressive but can still recur and produce metastases. ${ }^{4,5}$ IFPs are benign neoplasms with no risk or recurrence or metastasis after complete removal, but the identification of a common genetic defect implies a common oncogenetic pathway with GISTs. It is very interesting that Schildhaus et al actually report a case of simultaneously occurring IFP and GIST. $^{5}$ Despite these advances, the nature of the tumor cells remains a mystery and the origin of the submucosal mesenchymal progenitor cell that mutates to develop the tumor is still unknown.

Differential diagnosis is difficult even at a microscopic level, and comprehensive immunohistochemical analysis is employed to differentiate from other tumors such as GIST, mesenchymal sarcoma, schwannoma, and inflammatory myofibroblastic tumor. The latter is a rare mesenchymal neoplasm of the gut, positive to desmin, SMA, and Alk1 stains that is associated with locally invasive disease, lack of mitosesatypia, recurrence rates between 18 and 40\%, and a risk of malignant transformation. ${ }^{17}$ In our case, CD34 stain which is a commonly used marker of hematopoietic progenitor cells and endothelium was positive, as is in GISTs, but CD117 was negative. Vimentin stain, which is a marker of mesenchymal origin, was also positive.

It is not yet clear whether the classification of IFPs as a mesenchymal benign neoplasm has implications for treatment or follow-up, because in the vast majority of the reported cases, surgical excision alone was curative, and no reports exist of a malignant transformation. Because of the unknown yet origin of the lesion, the rarity of the disease in childhood and the relative absence of literature data for the

Table 1 Case reports of pediatric IFPs

\begin{tabular}{|l|l|l|l|l|l|l|}
\hline No. & Author & Year & Age $(\mathrm{y})$ & Sex & Gl location & Clinical features \\
\hline 1 & Samter $^{8}$ & 1966 & 4 & $\mathrm{M}$ & Colon & Pain, vomiting, perforation \\
\hline 2 & Samter $^{8}$ & 1966 & 8 & $\mathrm{~F}$ & Jejunum & Vomiting, diarrhea, anemia \\
\hline 3 & McGreevy et al $^{9}$ & 1967 & 2 & $\mathrm{~F}$ & lleum & Intussusception \\
\hline 4 & Persoff $^{8}$ & 1972 & 3 & $\mathrm{M}$ & lleum & Pain, vomiting, diarrhea \\
\hline 5 & Pollice $^{8}$ & 1984 & 8 & $\mathrm{M}$ & Rectum & Lower Gl bleeding, anemia \\
\hline $6-9$ & Blackshaw and Levison $^{6}$ & 1986 & $\mathrm{~N} / \mathrm{A}$ & $\mathrm{N} / \mathrm{A}$ & N/A & No details mentioned for pediatric cases \\
\hline 10 & Schroeder $^{8}$ & 1987 & 5 & $\mathrm{~F}$ & Stomach & Pain, vomiting, anemia \\
\hline 11 & Montgomery and Popek $^{10}$ & 1994 & $\mathrm{~N} / \mathrm{A}$ & $\mathrm{N} / \mathrm{A}$ & Ileum & Intussusception \\
\hline 12 & Dabral et al $^{11}$ & 2003 & 7 & $\mathrm{M}$ & Ileum & Intestinal obstruction \\
\hline 13 & Chongsrisawat et al $^{8}$ & 2004 & 4 & $\mathrm{~F}$ & Stomach & Anemia, fever \\
\hline 14 & Saiji et al $^{12}$ & 2006 & 15 & $\mathrm{M}$ & Ileum & Intussusception \\
\hline
\end{tabular}

Abbreviations: F, female; GI, gastrointestinal; IFP; Inflammatory fibroid polyp; M, male. 
disease, a cautious approach with periodic clinical surveillance of the affected children seems reasonable, with followup endoscopy reserved only for cases of endoscopically removed polyps.

\section{Conflict of Interest}

None.

\section{References}

1 Vanek J. Gastric submucosal granuloma with eosinophilic infiltration. Am J Pathol 1949;25(3):397-411

2 Lasota J, Wang ZF, Sobin LH, Miettinen M. Gain-of-function PDGFRA mutations, earlier reported in gastrointestinal stromal tumors, are common in small intestinal inflammatory fibroid polyps. A study of 60 cases. Mod Pathol 2009;22(8):1049-1056

3 Allibone RO, Nanson JK, Anthony PP. Multiple and recurrent inflammatory fibroid polyps in a Devon family ('Devon polyposis syndrome'): an update. Gut 1992;33(7):1004-1005

4 Huss S, Wardelmann E, Goltz D, et al. Activating PDGFRA mutations in inflammatory fibroid polyps occur in exons 12, 14 and 18 and are associated with tumour localization. Histopathology 2012; 61(1):59-68

5 Schildhaus HU, Cavlar T, Binot E, Büttner R, Wardelmann E, Merkelbach-Bruse S. Inflammatory fibroid polyps harbour mutations in the platelet-derived growth factor receptor alpha (PDGFRA) gene. J Pathol 2008;216(2):176-182
6 Blackshaw AJ, Levison DA. Eosinophilic infiltrates of the gastrointestinal tract. J Clin Pathol 1986;39(1):1-7

7 Kan H, Suzuki H, Shinji S, Naito Z, Furukawa K, Tajiri T. Case of an inflammatory fibroid polyp of the cecum. J Nippon Med Sch 2008;75(3):181-186

8 Chongsrisawat V, Yimyeam P, Wisedopas N, Viravaidya D, Poovorawan Y. Unusual manifestations of gastric inflammatory fibroid polyp in a child. World J Gastroenterol 2004;10(3):460-462

9 McGreevy P, Doberneck RC, McLeay JM, Miller FA. Recurrent eosinophilic infiltrate (granuloma) of the ileum causing intussusception in a two-year-old child. Surgery 1967;61(2):280-284

10 Montgomery EA, Popek EJ. Intussusception, adenovirus, and children: a brief reaffirmation. Hum Pathol 1994;25(2):169-174

11 Dabral C, Singh N, Singh PA, Misra V. Inflammatory fibroid polyp of small intestine in a child. Indian J Gastroenterol 2003;22(3):101

12 Saïji E, Ayadi-Kaddour A, Ben Slama S, et al. [Inflammatory fibroid polyp of the ileum presenting as intussusception: a case report in an adolescent]. Tunis Med 2006;84(7):454-457

13 Goldman RL, Friedman NB. Neurogenic nature of so-called inflammatory fibroid polyps of the stomach. Cancer 1967;20(1):134-143

14 Rubinstein R, Mogle P, Merguerian P, Rosenmann E. Inflammatory fibroid polyp of the small intestine: report of two cases and review of the literature. Isr J Med Sci 1983;19(9):828-833

15 Toole HJ, Moschopoulos AN. Eosinophilic granuloma of the gastrointestinal tract: report of two cases. Br J Surg 1959;46(199):445-448

16 Gönül II, Erdem O, Ataoğlu O. Inflammatory fibroid polyp of the ileum causing intussusception: a case report. Turk J Gastroenterol 2004;15(1):59-62

17 Ladd AP, Grosfeld JL. Gastrointestinal tumors in children and adolescents. Semin Pediatr Surg 2006;15(1):37-47 